\title{
Multiscale model for progressive damage prediction of carbon/epoxy laminates under thermal-mechanical loading
}

\author{
Wan-lei Liu*, Xin-long Chang, Xiao-jun Zhang, You-hong Zhang \\ Xi'an High-tech Institute, Xi'an 710025, China; \\ *vonleyliu@163.com
}

Keywords: Laser irradiation; Multiscale analysis; Bridge model; thermal-mechanical

\begin{abstract}
A multiscale model based bridge theory was proposed for the progressive damage analysis of carbon/epoxy laminates under couple laser and mechanical loading. The ablation model was adopted to calculate ablation temperature changing and ablation surface degradation. The polynomial strengthening model of matrix was used to improve bridging model for reducing parameter input. Thermal-mechanical analyses of the composite plate were performed using the ABAQUS/explicit program with the developed model implemented in the VUMAT. The simulation results showed that this model could be used to proclaim the mesoscale damage mechanism of composite laminates under coupled loading.
\end{abstract}

\section{Introduction}

Fiber reinforced composite materials are widely used for load carrying components in various fields such as aerospace, aviation, automotive, marine, and wind power industry. In particular, these materials have become indispensable in advanced aerospace and aeronautic structures. Carbon/epoxy composite materials present a high specific strength and stiffness, as well as superior fatigue properties. Although these traditional properties have been well investigated, the damage mechanics of composites structure induced by the coupled laser irradiation and mechanical loading have not been well understood $^{[1-3]}$.

Recently, with the laser weapon increasing rapidly in recent years, the behaviors of polymer composite materials under laser irradiation have attracted widely attention. Both experimentally and analytically methods have been established that composites show particular behavior under this type of action ${ }^{[4-7]}$. As previously reported, the alteration of structural performance is primarily result from two thermal effects, high temperature and mass loss due to surface ablation. Simultaneously, both stiffness and strength of composite exhibits a pronounced degradation. Obviously, these thermal loads play an important role in the change of constitutive relations of a laminate initially subjected to mechanical loads. In hence, it is necessary to develop a novel method for predicting composite damage under coupled laser irradiation and mechanical loading.

In the current study, a new multiscale model based bridge theory is proposed and applied for the progressive damage analysis of composite plates under coupled laser and thermal loading. The progressive damage model is implemented in the user material subroutine of the ABAQUS/explicit program, VUMAT, and used for the damage analysis of a composite plate. The macro- and microthermal-mechanical damage law and the ultimate strength of the composite plate under coupled laser and mechanical load are predicted by the proposed model.

\section{Modified bridge model}

\subsection{Ablation theory of composite plate under laser irradiation}

Since the heat exchange of carbon/epoxy composite is usually small and the laser irradiation time is short, the in-plane heat can be ignored. The thermal ablation analysis only aims at the thickness direction (as shown in Fig.1). Based on the different response of composite plate under laser irradiation, 
the ablation process is divided into three forms: heat exchange, matrix decomposition, carbon fiber sublimation.

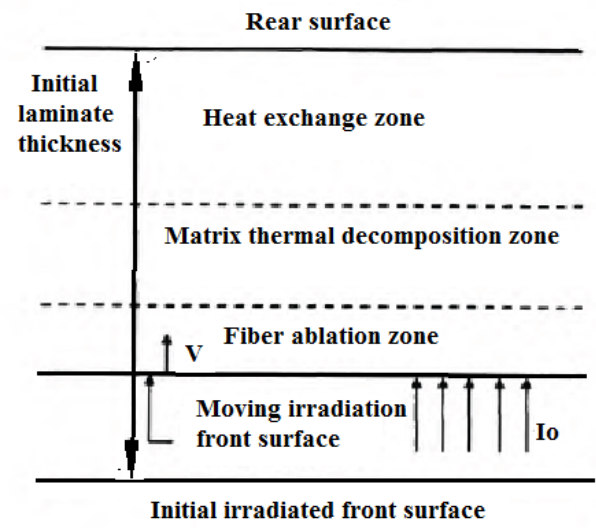

Fig.1 Ablation model

This heat exchange equation can be expressed as following:

$$
\frac{\partial}{x}\left(k \frac{\partial T}{\partial x}\right)+\rho c V \frac{\partial T}{\partial x}=\rho c \frac{\partial T}{\partial t}
$$

where $c, \rho, k, T, V$ represent the specific thermal capacity, density, conductivity parameter, temperature, and liner ablation speed respectively, and $\mathrm{T}$ is a function of coordinate $\mathrm{x}$ and time t. Surface irradiation energy follows the conservation law:

$$
\rho H_{s} V=a I_{0}+k \frac{\partial T}{\partial x_{i}}+I_{r}+I_{c}
$$

where $H_{s}, a, I_{0}, I_{r}, I_{c}$ are the effective ablation heat, energy absorption parameter, irradiation flow, thermal irradiation and heat convection loss.

The $I_{r}, I_{c}$ can be written as:

$$
\begin{gathered}
\left.I_{r}=\overline{\sigma \varepsilon} \mid T_{0}^{4}-T^{4}(0, t)\right] \\
I_{c}=h_{c}\left[T_{r}-T(0, t)\right]
\end{gathered}
$$

where $\bar{\sigma}, \bar{\varepsilon}, h_{c}, T_{0}, T_{r}, T(0, t)$ are the Boltzmann constant, surface irradiation parameter, convection parameter, irradiation environment temperature, air temperature of convection surface, and irradiation surface temperature, respectively. Because the irradiation and heat convection of composite underside surface is small, it can be considered as natural heat transfer face.

\subsection{Bridge model}

Based on the bridge theory ${ }^{[8-10]}$, in a general three-dimensional stress state, the internal stress of fiber and matrix in a representative volume element (RVE) of the lamina satisfy

$$
\left\{d \sigma_{i}^{m}\right\}=\left[A_{i j}\right]\left\{d \sigma_{j}^{f}\right\}
$$

Using the above bridge matrix $\left[\mathrm{A}_{\mathrm{ij}}\right]$, following equations can be derived

$$
\begin{gathered}
\left\{d \sigma_{i}^{f}\right\}=\left(V_{f}[I]+V_{m}\left[A_{i j}\right]\right)^{-1}\left\{d \sigma_{j}\right\}=\left[B_{i j}\right]\left\{d \sigma_{j}\right\} \\
\left\{d \sigma_{i}^{m}\right\}=\left[A_{i j}\right]\left(V_{f}[I]+V_{m}\left[A_{i j}\right]\right)^{-1}\left\{d \sigma_{j}\right\} \\
{\left[S_{i j}\right]=\left(V_{f} \mid S_{i j}^{f}\right]+V_{m}\left[S_{i j}^{m}\left[A_{i j}\right]\left[B_{i j}\right]\right.}
\end{gathered}
$$

where $\left\{d \sigma_{j}\right\}=\left\{d \sigma_{11}, d \sigma_{22}, d \sigma_{12}\right\}^{T}$ are the overall applied stress increments.. By means of bridge model and the external load applied in the composite plate, the equilibrium equation can be derived as 


$$
\left\{\begin{array}{l}
d N_{x x} \\
d N_{y y} \\
d N_{x y} \\
d M_{x x} \\
d M_{y y} \\
d M_{x y}
\end{array}\right\}=\left\{\begin{array}{llllll}
Q_{11}^{I} & Q_{12}^{I} & Q_{13}^{I} & Q_{11}^{I I} & Q_{12}^{I I} & Q_{13}^{I I} \\
Q_{12}^{I} & Q_{22}^{I} & Q_{23}^{I} & Q_{12}^{I I} & Q_{22}^{I I} & Q_{23}^{I I} \\
Q_{13}^{I} & Q_{23}^{I} & Q_{33}^{I} & Q_{13}^{I I} & Q_{23}^{I I} & Q_{33}^{I I} \\
Q_{11}^{I I} & Q_{12}^{I I} & Q_{13}^{I I} & Q_{11}^{I I I} & Q_{12}^{I I I} & Q_{13}^{I I I} \\
Q_{12}^{I I} & Q_{22}^{I I} & Q_{23}^{I I} & Q_{12}^{I I I} & Q_{22}^{I I I} & Q_{23}^{I I I} \\
Q_{13}^{I I} & Q_{23}^{I I} & Q_{33}^{I I} & Q_{13}^{I I I} & Q_{23}^{I I I} & Q_{33}^{I I I}
\end{array}\right\}\left\{\begin{array}{c}
d \varepsilon_{x x}^{0} \\
d \varepsilon_{y y}^{0} \\
2 d \varepsilon_{x y}^{0} \\
d k_{x x}^{0} \\
d k_{y y}^{0} \\
2 d k_{x y}^{0}
\end{array}\right\}
$$

$\mathrm{N}$ is the total number of lamina plies in the laminate and $\left(C_{i j}^{L}\right)_{k}$ is the stiffness elements of the $k$ th lamina in the global coordinate system. In Eq.(7), $d N_{x x}, d N_{y y}$, and $d N_{x y}$ and $d M_{x x}, d M_{y y}$, and $d M_{x y}$ are the overall incremental in-plane forces and moments per unit length exerted on the laminate, respectively.

\subsection{Polynomial strengthening model of matrix}

Ramberg-Osgood model is a continuum nonlinear constitutive model and widely used in many elastic-plastic materials. It can be expressed as

$$
\varepsilon=\frac{\sigma}{E}+a\left(\frac{\sigma}{b}\right)^{n}
$$

where $a, b$ and $n$ are the material constants. Though this model doesn't define the yield point of the material, the initial slope value of curve is taken as the Young modulus E.

With the stress increasing, the slope of curve is decreased. From the Ramberg-Osgood model, it is known that the matrix strain can be expressed as stress polynomial equation. Hence, the stress-strain relationship can be assumed as

$$
\varepsilon=A \sigma+B \sigma^{2}+C \sigma^{3}+D \sigma^{4}
$$

where $A, B, C, D$ are the parameters related to material and can be attained by experiment.

The stress-strain curve of matrix is presented as an example in Fig.2. On the basis of Eq.(9), the coefficient can be obtained. If the stress state is known, the equivalent modulus of matrix can be derived.

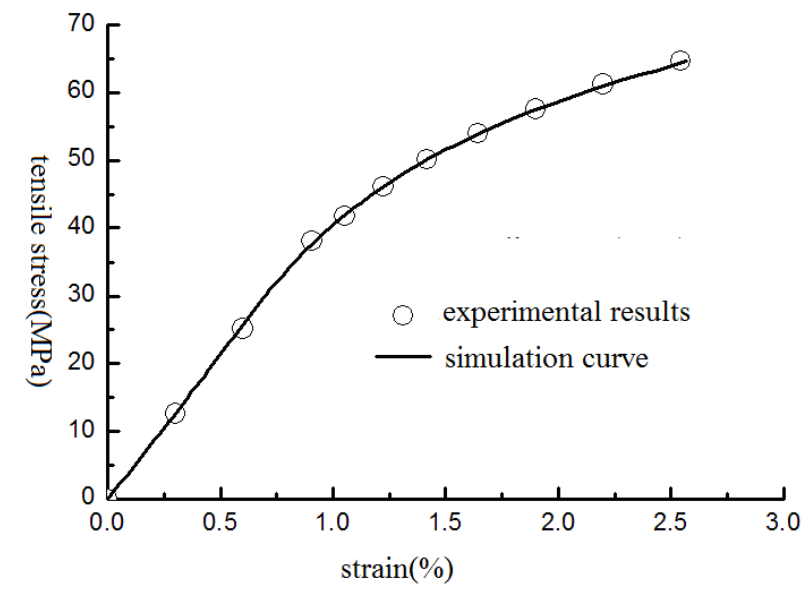

Fig.2 The polynomial strengthening curve of matrix

\section{Damage analysis of carbon/epoxy laminates under coupled laser and tensile loading}

\subsection{Finte element modle}

Thermal-mechanical response of composite material is simulated by ABAQUS/Explicit using the developed bridge model implemented in VUMAT. The material used in simulation is AS4/3501-6 and 
the stacking sequence is $\left[ \pm 45 / 90 / 0_{4} / \pm 45 / 0_{2}\right]_{S}$. The fiber volume fraction is 0.62 and plate thickness is $2.54 \mathrm{~mm}$. All specimens are $304.8 \mathrm{~mm}$ length and $25.4 \mathrm{~mm}$ width, as shown in Fig.3(a). The input properties used in simulation are taken from Ref[4,5]. For the symmetry of composite plate, quarter model is used in simulation analysis.

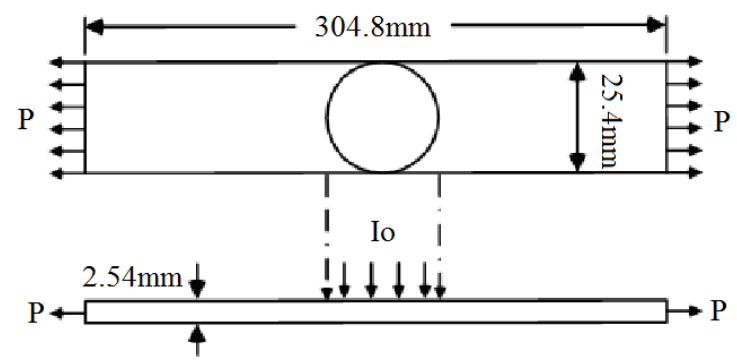

Fig.3 Thermal-mechanical FE model of composite plate

The carbon fiber and matrix properties can be derived through reverse calculation. Notice there only consider the stiffness and strength in fiber's axial direction changed with the temperatures. The bridge parameters are $\alpha=\beta=0.45$ 。For the matrix in composite is not the main supporting constituent, so the thermal response of matrix can be divided into four temperature sections, each temperature point is $20^{\circ} \mathrm{C}, 150^{\circ} \mathrm{C}, 200^{\circ} \mathrm{C}, 3316^{\circ} \mathrm{C}$. As the matrix appears thermal decomposition at the $200^{\circ} \mathrm{C}$ $\sim 3316^{\circ} \mathrm{C}$, so the stiffness and strength of matrix possess the infinite value. The polynomial strengthening equations of matrix can be fitted using the equations shown in Table 1 and the fiber properties was shown in Table 2.

Table 1 Polynomial strengthening equations of matrix

\begin{tabular}{cc}
\hline temperature $/{ }^{\circ} \mathrm{C}$ & Four order polynomial fitting \\
\hline 20 & $\varepsilon=2.381 E^{-4} \sigma+6.942 E^{-7} \sigma^{2}-7.718 E^{-8} \sigma^{3}+1.619 E^{-9} \sigma^{4}$ \\
150 & $\varepsilon=5.338 E^{-4} \sigma+1.334 E^{-6} \sigma^{2}-3.100 E^{-7} \sigma^{3}+1.472 E^{-8} \sigma^{4}$ \\
200 & $\varepsilon=5.760 E^{-4} \sigma+3.198 E^{-5} \sigma^{2}-3.392 E^{-6} \sigma^{3}+1.112 E^{-7} \sigma^{4}$ \\
\hline
\end{tabular}

Table 2 Mechanical properties of AS4 carbon fiber

\begin{tabular}{cccccccc}
\hline \multirow{2}{*}{ Temperature } & $E_{11}^{f} /$ & $E_{22}^{f} /$ & $G_{12}^{f}$ & $v_{12}^{f}$ & $a_{1}^{f} / 10^{-6} /{ }^{\circ} \mathrm{C}$ & $a_{2}^{f} / 10^{-6} /{ }^{\circ} \mathrm{C}$ & $X_{t}^{f} / \mathrm{MPa}$ \\
& $\mathrm{GPa}$ & $\mathrm{GPa}$ & $/ \mathrm{GPa}$ & & 15 & 15 & 2660 \\
\hline $20{ }^{\circ} \mathrm{C}$ & 220 & 15 & 15 & 0.2 & -0.5 & 15 & 2660 \\
$200^{\circ} \mathrm{C}$ & 220 & 15 & 15 & 0.2 & -0.5 & 15 & 532 \\
$740^{\circ} \mathrm{C}$ & 220 & 15 & 15 & 0.2 & -0.5 & 15 & 532 \\
$3316^{\circ} \mathrm{C}$ & 220 & 15 & 15 & 0.2 & -0.5 & \\
\hline
\end{tabular}

\subsection{Tensile failure}

The ultimate strengths of composite plate at different instances are shown in Table 3. The maximum tensile strength of initial specimen is 966.7 MPa according with the data in Ref.[4].

Table 3 Simulation results of tensile strength

\begin{tabular}{ccccc}
\hline \multirow{2}{*}{$\begin{array}{c}\text { Laser power } \\
\text { density/kw/cm }\end{array}$} & Irradiation time/s & \multicolumn{3}{c}{ Tensile strength } \\
\cline { 3 - 5 } & & $\begin{array}{c}\text { Simulation } \\
\text { results/MPa }\end{array}$ & $\begin{array}{c}\text { Experimental } \\
\text { results /MPa }\end{array}$ & Error/\% \\
\hline 0 & 0 & 952.8 & 979.3 & 2.7 \\
1.4 & 4.0 & 470.3 & 489.7 & 4.0 \\
1.5 & 3.7 & 468.5 & 489.7 & 8.2 \\
2.45 & 8.0 & 224.5 & 244.8 & 6.7 \\
\hline
\end{tabular}

When the laser intensity keeps constant, the maximum strength of composite plate decre- ases with the time increasing and the decrease rates are related with the laser intensity. While the laser intensity being $2.5 \mathrm{kw} / \mathrm{cm}^{2}$, matrix and fibers are ablation. At the same time, the irradiation face widens rapidly. When the laser intensity is $2.5 \mathrm{kw} / \mathrm{cm}^{2}$ and irradiation time are $0.5 \mathrm{~s}, 1.0 \mathrm{~s}$, the outerlamina is ablation, 
but this mode has little influence on the axial strength. As the irradiation time increasing, the number of ablation layers is also increased and the major bearing capability of composite is lost.

\subsection{Damage mechanism analysis}

In this simulation, $0^{\circ}$ laminas are the main load-carrying structure and the composite strength is determined by the fiber's condition. To better analyze the damage mechanism of composite under coupled loadings, thickness directional temperature distribution and fiber strength-temperature curves are plotted in one picture, as shown in Fig.4.

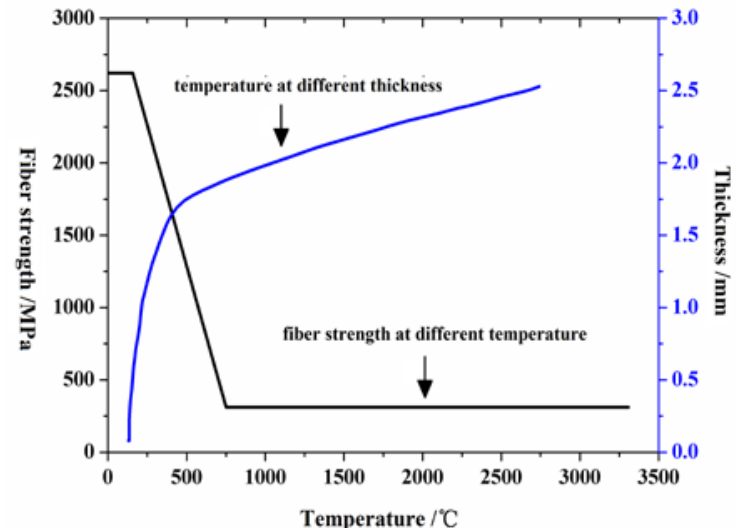

(a) $\mathrm{I}_{0}=8.0 \times 10^{-3} \mathrm{kw} / \mathrm{mm}^{2}, \mathrm{t}=4.0 \mathrm{~s}$

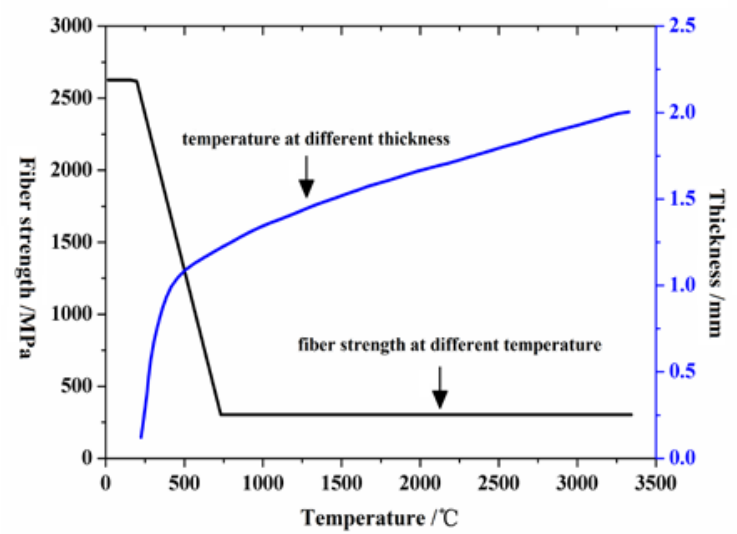

(c) $\mathrm{I}_{0}=15 \times 10^{-3} \mathrm{kw} / \mathrm{mm}^{2}, \mathrm{t}=8.0 \mathrm{~s}$

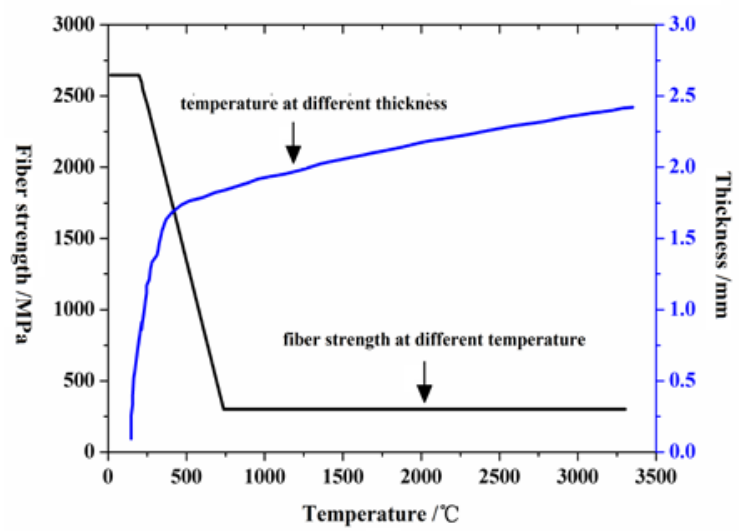

(b) $\mathrm{I}_{0}=14.0 \times 10^{-3} \mathrm{kw} / \mathrm{mm}^{2}, \mathrm{t}=3.7 \mathrm{~s}$

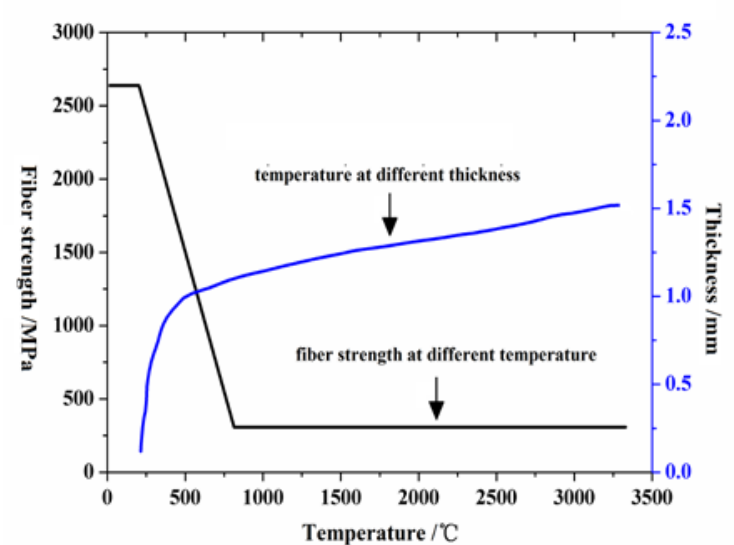

(d) $\mathrm{I}_{0}=24.5 \times 10^{-3} \mathrm{kw} / \mathrm{mm}^{2}, \mathrm{t}=5.0 \mathrm{~s}$

Fig. 4 Temperature-thickness and fiber strength-temperature curves

As shown in Fig.4(a), composite ablation does not occurred and the fiber strength of laminas which coordination lower than $1.0 \mathrm{~mm}$ are keep unchanged. The fiber strength of laminas coordination larg- er than $1.8 \mathrm{~mm}$ keeps at the lower stage (532MPa). Fiber strength of laminas between $1.0 \mathrm{~mm}$ and $1.8 \mathrm{~mm}$ is gradually decreasing. In this case, the composite tensile strength decreases $50 \%$ though the fib- er is not ablation. The main reason is matrix decomposition under high temperature induced by laser irradiation.

It is only occurred composite ablation at 1th lamina in Fig.4(b). Matrix decomposition is still the main reason for composite strength decrease in this case. In Fig.4(c) and Fig.4(d), the 1-4 th laminas are ablation and the residual thickness of irradiation region is $2.0 \mathrm{~mm}$. Composite strength is $25 \%$ remains after laser irradiation. The reasons leading to this phenomenon can be divided into two categories. One is the fiber ablation induces the composite thickness loss and the other one is temperature increasing through composite thickness direction causes the fiber strength degradation.

\section{Summary}

In the present study, thermal characteristic and progressive damage of carbon/epoxy composite plate under coupled laser-mechanical loading is analyzed using explicit element method together with developed bridge model. 
Based on improved bridging model, tensile strength of the laminate under various laser intensity and irradiation time is calculated. The effects of different laser intensity and irradiation time on the laminate tensile strength and mesoscale damage mechanism are analyzed. The predicted tensile strength has a good agreement with experimental results. Epoxy matrix thermal decomposition and the carbon fiber ablation are the main damage modes of composite plate under laser irradiation. These two damage modes can both induce the composite strength decrease. While the fiber ablation can absorb amount of heat and can reverse decrease the laser irradiation influence efficiency to the composite strength. Taken together, it is suggested that the current numerical model can be improved to analyze the progressive damage and ultimate strength of composite structures.

\section{References}

[1] Patton R.D, Pittman C.U.J, Wang L, et al. Ablation, mechanical and thermal conductivity properties of vapor grown carbon fiber/phonetic matrix composites. Composites: Part A,2002,33:243-251.

[2] Wan H, Hu K.W, Mu J.Y, et a1. Damage analysis of fiber reinforced resin matrix composites irradiated by CW laser[J]. High Power laser and particle beams,2008,20(1):96-101.

[3] Spottswood S.M. Progressive failure analysis of a composite shell. Composite Structure, 2001(53):117-131.

[4] Chen J.K, Sun C.T, Chang C I. Failure analysis of a graphite/epoxy laminate subjected to combined thermal and mechanical loading. J Composite Materials, 1985, 19: 427-442.

[5] Wagner H.D. Thermal residual stress in composites with anisotropic interphases. Physical Review B, 1996, 53(9): 5055-5058.

[6] Chen J.K, Perea A, Allahdadi F.A. A Study of Laser/Composite Material Interactions. Composites science and Technology,1995(54):35-44.

[7] Dimitrienko Y.I. Thermechanical Behavior of Composite Materials and Structures under High Temperature. Structures Composites, 1997(28)A:453-471.

[8] Huang Z.M. A Modified Ultimate Failure Criterion and Material Degradation Scheme in Bridging Model Prediction for Biaxial Strength of Laminates. Journal of Composite Materials, 2008 42(20):2123-2141.

[9] Huang Z.M. A bridging model prediction of the ultimate strength of composite laminates subjected to biaxial loads. Composite Science and Technology, 2004(64): 395-448.

[10] Huang Z M. Inelastic and failure analysis of laminate structures by ABAQUS incorporated with a general constitutive relationship. Journal of Reinforced Plastics and Composites, 2007, 26(11): $1135-1181$. 